\title{
Impact of Antifibrotic Treatment on the Course of Schistosoma mansoni Infection in Murine Model
}

\author{
Michal Giboda ${ }^{+}$, James M Smith*
}

San Juan Bautista School of Medicine, GPO Box 71365, San Juan, Puerto Rico 00936-8465 *Institute of

Parasitology, McGill University, 21111 Lakeshore Road, Ste Anne de Bellevue, Canada, PQ H9X 3V9

\begin{abstract}
Administration of an antifibrotic agent as an adjunct to antihelmintic treatment with the objective of morbidity reduction was investigated in the murine schistosomiasis mansoni model. Antifibrotic, $\beta$ aminopropionitrile treatment has a profound effect on the cellular matrix composition of the liver granuloma of Schistosoma mansoni infected mice when given alone, resulting in increase macrophage infiltration. These macrophages, in response to stimulation with soluble egg antigen or lipopolysaccharide produced elevated levels of nitric oxide but low levels of tumor necrosis factor alpha compared to untreated infected mice. This also correlated with reduced liver granuloma size. In spite of low numbers of eggs in the liver, mice receiving a combine treatment had a high level of resistance to a challenge infection compared with mice receiving only praziquantel. Those mice also exhibited a reduced lymphocyte proliferative response, similar to that of infected untreated mice. Antifibrotic treatment has an impact on the dynamic of the cellular nature of granulomas and impacts on the host immunity to infection.
\end{abstract}

Key words: Schistosoma mansoni - $\beta$-aminopropionitrile - antifibrotic treatment - granulomas cells - cytokines lymphocytes proliferation - resistance

Liver and intestinal fibrosis is a major sequel to granulomatous schistosomiasis mansoni mostly responsible for portal hypertension, formation of esophageal varices, and intestinal bleeding of infected human and mice. Fibrogenesis is a dynamic, potentially preventable process comprised of many biological steps carefully controlled by sequential action of stimulatory and inhibitory signals produced by macrophages, lymphocytes and fibroblast such as transforming growth factor beta (TGF- $\beta$ ), cytokines and fibrogenic mediators (Wyler 1992, Gressner 1992, Wynn et al. 1993).

Many approaches were applied in order to prevent or decreased liver fibrosis in schistosomiasis mansoni infections. Intensive research in the area of immunological intervention brought many promising results for the limitation of extended fibrosis (Czaja et al. 1989, Cheever et al. 1994, 1995, Lukacs et al. 1994, Wynn et al. 1995) but anthelmintic chemotherapy is still the only appli-

\footnotetext{
These investigations received financial assistance from the UNDP/World Bank/WHO Special Programme for Research and Training in Tropical Diseases, from San Juan Bautista School of Medicine, and from the Medical Research Council of Canada.

${ }^{+}$Corresponding author. Fax: +787-746-3093. E-mail: giboda@caribe.net

Received 16 April 1997

Accepted 30 June 1997
}

cable treatment for liver scars in infected persons. A recent study has demonstrated that chemotherapy with praziquantel (PZQ), which, by killing the mature eggs, removes the source of antigenic stimulation, can stabilize collagen deposition. However, with the interruption of immune modulation by PZQ, which normally occurs in a chronic infection, the prognosis for reabsorption of fibrotic scarring is poor (Kresina et al. 1994). Controversial data from field studies on the impact of antiparasitic treatment on resolution of liver fibrosis stimulated the search for other options. Antifibrotic chemotherapy as an adjunct to anthelmintic chemotherapy was proposed as a possible therapeutic modality (Wyler 1992).

Granulomas elicited by Schistosoma mansoni eggs are constituted by mesenchymal, myeloid and lymphoid cells with organ dependent differences in cellular composition of the granulomas and the relative amounts and types of collagen they contain (Weinstock \& Boros 1983, Grimaud et al. 1987, Hirata et al. 1993). Both fibrillar collagen, types I and III, represent $80 \%$ and $15-20 \%$, respectively, of the total collagen synthesized by fibroblast (Kovacs 1991) and both dominate the periovular granuloma (Grimaud et al. 1987). A critical extracellular modification of newly synthesized collagen is lysyl hydroxylysyl oxidation, which results in the formation of cross-linkages between the alpha chains of adjacent molecules, and is the 
basis of the structural stability and tensile strength of collagen. In murine schistosomiasis, studies on collagen cross-linking have shown that (a) the transcription rate of lysyl oxidase increases 5-fold, 8 weeks after infection, and is paralleled by an upregulation in the transcription of $\alpha$ (1) I procollagen which rose to maximum level at 8 weeks p.i.; (b) myofibroblasts, which represent the main cell population in the peripheral area of the granuloma, are involved in the expression of lysyl oxidase; (c), that this enzyme is not found in hyalinized fibrotic tissue (Sommer et al. 1993), and (d) pyridinolin, a cross-linking amino-acid, found in mature collagen, shows a 12-fold increase in concentration in granulomatous tissues by 25 weeks p.i. (Ricard-Blum et al. 1992). It has also been demonstrated that a novel fibrogenic cytokine, fibrosin, which is produced by CD4 lymphocytes, mast cells and fibroblast acts in an autocrine fashion causing autonomous over expression of collagen (Wyler 1995).

It is possible to pharmacologically affect the post-translational steps of collagen cross-linking with the lathyrogenic agent $B$-aminopropionitrile (BAPN), which irreversibly inhibits lysyl oxidase (LO) by decrease of LO mRNA expression (Yeowell et al. 1994). The level of the $\alpha 1$ (I) collagen mRNA was not affected by BAPN. No significant change was observed in the steady-state level of LO mRNA in fibroblasts isolated from patients with certain genetic connective tissue disorders (Yeowell et al. 1994). A substantial body of literature presents evidence on the beneficial effect of BAPN for the prevention of scar formation in humans (Peacock \& Madden 1966, Keiser \& Sjoerdsma 1967).

\section{BAPN in Murine Schistosomiasis Mansoni}

With the objective of morbidity reduction, we have used PZQ, in conjunction with the antifibrotic agent BAPN, in the murine model of schistosomiasis mansoni (Giboda et al. 1992, 1994, 1995). In order to prevent cross-linking of newly deposited collagen, BAPN administration commenced at the time of egg deposition in the tissues, with a daily dosage of $5 \mathrm{mg}$ per mice for following 20 days. The continuity of the egg laying was interrupted by a double dose of PZQ, 9 days apart, with the intention of killing all the eggs in the tissues. Since PZQ has the capacity to kill only mature eggs, a single dose of PZQ would leave maturing eggs unharmed and allow them to complete their development (Giboda \& Zdarska 1994, Giboda \& Smith 1994). This may be a reason why many authors, after giving a single treatment with PZQ, report that there is a delay before a substantial decrease in the level of collagen in the granulomas is observed.
In order to prevent cross-linking of newly laid collagen, all experimental animals were autopsied the day following the last dose of BAPN. Mice were examined using quantitative parasitological criteria and several immunological markers. The details of experimental designs are published elsewhere (Giboda et al. 1992, 1994, 1995).

\section{RESULTS}

The experimental data presented here originated from two main groups of animals: from mice in which the infection was patent throughout the entire experiment (i.e. those untreated or treated with BAPN, which is not curative to $S$. mansoni infection); and those in which the infection was terminated with anthelmintic (those treated with PZQ alone or BAPN+PZQ) and results from these groups should be considered to be a reflection of the post-treatment state of the host (Figs 1, 2, 3).

Quantitative parasitological criteria - Depending on the treatment group (PZQ alone, BAPN alone, $\mathrm{PZQ}+\mathrm{BAPN}$ ), organ specific effects were observed using microscope image analysis (Fig. 1). Most notable were: the relatively small size of granulomas in the livers of BAPN treated mice $(\mathrm{P}<0.0008)$, and the relatively large size and irregular shape of the granulomas in the intestinal tissues $(\mathrm{P}<0.02)$ compared with those in control. In contrast, the combine therapy (BAPN+PZQ) led to smaller intestinal granulomas $(\mathrm{P}<0.00002)$ than those in the control, and liver granulomas, although highly variable, had mean size similar to those in the controls. Mice treated with the combination of

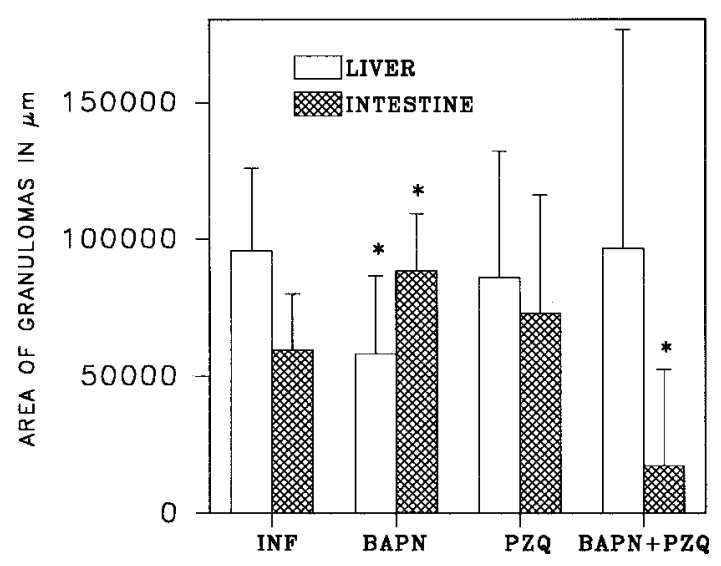

Fig. 1: microscope image analysis of liver and intestinal egg granuloma size. The size of the granulomas varied between groups of mice depending on the site and treatment. In BAPN ( $\beta$-aminopropionitrile) treated mice liver granuloma were significantly smaller than those from untreated controls but those in the intestine were significantly larger. Intestinal granuloma in the combine treated mice $(\mathrm{BAPN}+\mathrm{PZQ})$ (praziquantel) were significantly reduced in size $(* \mathrm{P}<0.05)$, significantly different from untreated infected controls (INF) at the level $\mathrm{P}<0.05$. 
BAPN+PZQ had decreased liver and spleen weights, and there was a reduction in the number of eggs trapped in both liver and intestine, compared with untreated mice and those given PZQ alone.

A microscopic examination of the granulomas could not distinguish a difference between the collagen in granulomas from mice treated with BAPN and the collagen in the granulomas of mice infected and non-treated. Antifibrotic treatment (BAPN only) initiated an early onset of egg excretion and a higher egg output when compared with untreated controls. Post treatment, egg excretion terminated sooner when mice were treated with BAPN+PZQ than in mice treated with PZQ alone only.

Post treatment resistance - Since the resistance to reinfection is directed at immature worms, and depends on egg deposition and related pathology in the liver (Wilson 1990), it was of particular interest to investigate the post treatment resistance to a secondary challenge in the mice treated with BAPN+PZQ, which had a low liver egg load. Naive mice, and those infected and treated with $\mathrm{BAPN}+\mathrm{PZQ}, \mathrm{BAPN}$ alone or PZQ alone, were challenged 2 and 5 weeks post-treatment. Mice receiving a combined antifibrotic and anthelmintic treatment $(\mathrm{BAPN}+\mathrm{PZQ})$ retained a high level of resistance, similar to that observed in untreated infected mice. On the other hand, mice receiving only PZQ were as susceptible to challenge as were uninfected control mice. The mechanisms responsible for the observed post-treatment resistance to challenge in mice receiving combine $\mathrm{BAPN}+\mathrm{PZQ}$ could not be correlated with either liver egg load and subsequently altered portal blood flow, or to peritoneal macrophages activation, and suggests the probability of an immunologically based resistance similar to that induced by radiation-attenuated cercariae. Additional histological observations have indicated that granulomas from BAPN+PZQ treated mice contain a greater number of inflammatory cells within the matrix of granulomas, compared with granulomas from untreated mice or treated with PZQ alone. This might suggest an increase in local concentration through proliferation, and/or increased migration of immunologically active cells into granulomas.

Immune response modulated by antifibrotic treatment - The combine anthelmintic and antifibrotic treatment resulted in profound changes to the liver granuloma cell matrix and the composition and function of the cellular infiltrate (Fig. 2). Although granulocytes always predominated in the infiltrate, the proportion of the cells which were macrophages $(M \phi)$ was higher in mice treated with BAPN alone (39.2\%) than in infected mice which were untreated (15.2\%) or treated with PZQ alone
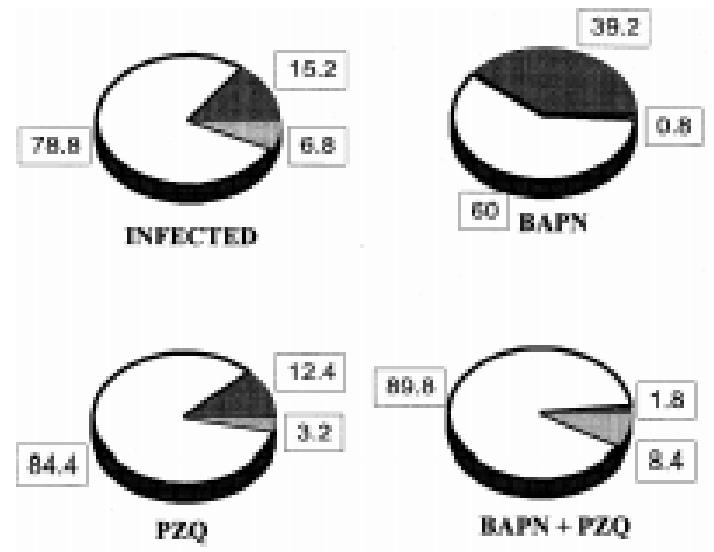

(12.4\%), and much higher than in mice given the combine treatment (1.8\%). Morphologically all the lymphocytes appeared to be at a state of rest.

Two products associated with $\mathrm{M} \phi$ activation and cytotoxicity (TNF- $\alpha$ and NO) were only detected in mice harboring a patent infection (Fig. 3). Granuloma M $\phi$ from both infected non-treated mice and those treated with BAPN, showed a small increase in TNF- $\alpha$ production in response to soluble egg antigen (SEA) as compared with nonstimulated cells. The response was more pronounced to LPS (lipopolysacharide), particularly by $\mathrm{M} \phi$ derived from the liver granulomas of infected non-treated mice. The TNF- $\alpha$ concentrations produced by M $\phi$ of the BAPN-treated mice, in response to LPS, were about 50\% lower than those produced by $\mathrm{M} \phi$ from untreated infected mice. The lower level of TNF- $\alpha$ produced by granuloma macrophages from BAPN treated mice is coincident with the reduced granuloma size observed in these animals. Joseph and Boros (1994) have shown that depletion of TNF- $\alpha$ with anti-TNF- $\alpha$ antibodies results in smaller granulomas, probably through TNF- $\alpha$ induced ICAM-1 expression (Lukacs et al. 1994). It has previously been shown that TNF- $\alpha$ up regulates adhesion molecules that participate in cellular recruitment and lymphocyte activation (Lukacs et al. 1994). Pro-adhesive effect of TNF$\alpha$ by enhancing the binding capacity of ECM-specific $\beta_{1}$ integrins expressed on surface of $\mathrm{T}$ cells was already reported (Gilat et al. 1996). 

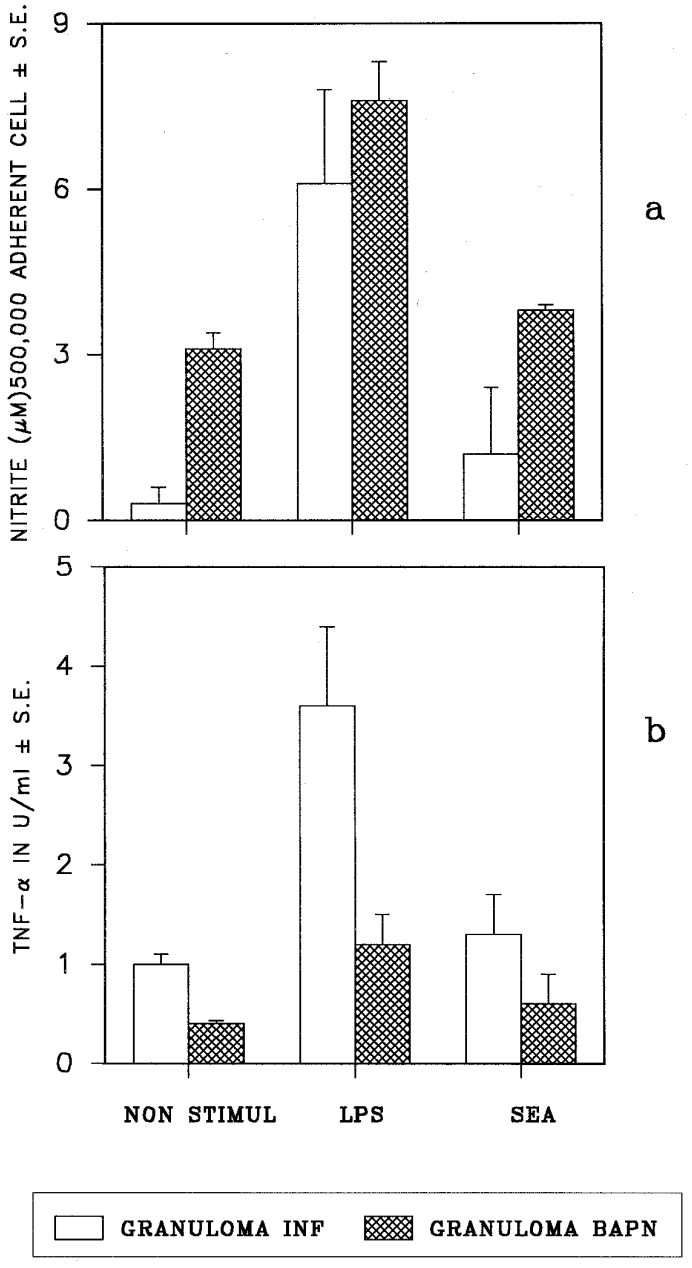

Fig. 3: (a) nitrite and (b) TNF- $\alpha$ (tumor necrosis factor alpha) production by non stimulated (NON STIMUL) granuloma macrophages or by those stimulated with lipopolysacharide (LPS) or soluble egg antigen (SEA). These two products, associated with activated macrophages, were only detected in mice with patent Schistosoma mansoni infections (see text for details).

Macrophages from BAPN-treated mice produced elevated levels of NO, either when stimulated with SEA or LPS, or in the presence of culture medium only, in comparison with cells from untreated mice (Fig. 3). In the case of cells stimulated with SEA antigen, the differences were statistically significant $(\mathrm{P}<0.05)$. It is difficult to assess the role of NO in resistance to post treatment challenge. Although infected mice after combined PZQ+BAPN treatment displayed a much higher level of post treatment resistance to reinfection (90\% two weeks post treatment and $74 \%$ five weeks p.t), the BAPN treated group was not tested since resistance would be expected in the presence of a patent infection. Future in vivo studies in which NO production could be inhibited, may give some insight into the relative role of this molecule in post treatment resistance.

The proliferative response of granulomas or spleen derived lymphoid cells to SEA and ConA stimulation was lower in untreated and $\mathrm{BAPN}+\mathrm{PZQ}$ treated groups than in those from BAPN or PZQ treated mice (data not showed). This is of particular interest as both groups (infected and $\mathrm{BAPN}+\mathrm{PZQ}$ ) showed high post treatment resistance. The cellular response that regulates protective immunity is still unresolved. A negative association has been reported in older (14-35 years) but not younger (9-13 years) individuals between lymphocyte proliferation responses to adult worm or schistosomulum antigens and subsequent reinfection (Roberts et al. 1993). The reduced proliferative response to SEA was associated with smaller lung granulomas in mice (Lukacs et al. 1994). In contrast, we found the greater proliferative response $(\mathrm{P}<0.05)$ by lymphocytes from liver granulomas from BAPN treated mice was associated with significantly smaller granulomas (Fig. 1), compared to all other experimental groups. It is unlikely that BAPN is directly responsible for increased proliferation as studies on rat hepatocytes have shown that it does not effect the rate of DNA synthesis (Ikeda et al. 1994). The differences observed in the host/parasite interaction which are dependent on the state of granuloma collagen crosslinking has to be investigated further in order to elucidate the underlying mechanisms involved.

A picture of the schistosoma egg granuloma is emerging as one which encompasses complex and intricate cell to cell interactions, involving a variety of adhesion molecules and orchestrated by multi-tasking cytokines. It is evident that modulation of this phenomenon by drug intervention, such as antifibrotic treatment preventing cross-linking of newly synthesize collagen, can have a significant impact on the pathological profile of the disease. It is clear that careful analysis of the presence and temporal patterns of these interacting molecules are required to fully understand and predict the outcomes of chemotherapy.

\section{REFERENCES}

Cheever AW, Williams ME, Wynn TA, Finkelman FD, Seder RA, Cox TM, Hieny S, Caspar P, Sher A 1994. Anti-IL-4 treatment of Schistosoma mansoni-infected mice inhibits development of $\mathrm{T}$ cells and non$\mathrm{B}$, non-T cells expressing Th2 cytokines while decreasing egg-induced hepatic fibrosis. I Immunol 153: 753-759.

Cheever AW, Finkelman FD, Cox TM 1995. Antiinterleukin-4 treatment diminishes secretion of Th2 cytokines and inhibits hepatic fibrosis in murine 
schistosomiasis japonica. Parasite Immunol 17: 103109.

Czaja MJ, Weiner FS, Takahashi S, Giambrone MA, van der Meide PH, Schellekens H, Biempica L, Zern MA 1989. $\gamma$-interferon treatment inhibits collagen deposition in murine schistosomiasis. Hepatology 10: 795-800.

Giboda M, Smith JM 1994. Schistosoma mansoni eggs as target for praziquantel: efficacy of oral application in mice. J Trop Med Hyg 97: 98-102.

Giboda M, Zdarska Z 1994. Alkaline phosphatase as marker of Schistosoma mansoni egg viability. Folia Parasitolol (Praha) 41: 55-58.

Giboda M, Smith JM, Prichard RK 1994. Reduction in tissue egg load and maintenance of resistance to challenge in mice infected with Schistosoma mansoni, following combined treatment with praziquantel and an antifibrotic agent. Ann Trop Med Parasitol 88: 385-395.

Giboda M, Jacobs P, Smith JM, Miller SC 1995. Immune response of mice infected with Schistosoma mansoni is modulated by antifibrotic treatment. Ann Trop Med Hyg 89: 415-424.

Giboda M, Zenka J, Julis I, Vitovec J 1992. Experimental schistosomiasis mansoni: modulation of granulomas by inhibition of collagen cross-link formation. Preliminary report. Ann Trop Med Parasitol 86: 631636.

Gilat D, Cahalon L, Hershkoviz R, Lider O 1996. Interplay of $\mathrm{T}$ cells and cytokines in the context of enzymatically modified extracellular matrix. Immunol Today 17: 16-20.

Gressner AM 1992. Hepatic fibrogenesis: the puzzle of interacting cells, fibrogenic cytokines, regulatory loops, and intracellular matrix molecules. $Z$ Gastroenterol 30 (Suppl. 1): 5-16.

Grimaud JP, Boros DL, Takiya C, Mathew RC, Emonard $\mathrm{H}$ 1987. Collagen isotypes, laminin, and fibronectin in granulomas of the liver and intestine of Schistosoma mansoni-infected mice. Am J Trop Med Hyg 37: 335-344.

Hirata M, Takushima M, Kage M, Fukuma T 1993. Comparative analysis of hepatic, pulmonary, and intestinal granuloma formation around freshly laid Schistosoma japonicum eggs in mice. Parasitol Res 79: 316-321.

Ikeda H, Ogata I, Fujiwara K 1994. Evidence that impaired intracellular collagen synthesis reduces proliferation in cultured rat hepatocytes. Biochem Biophys Res Commun 200: 1701-1707.

Joseph AL, Boros DL 1993. Tumor necrosis factor plays a role in Schistosoma mansoni egg-induced granulomatous inflammation. J Immunol 151: 5461-5471.

Keiser HR, Sjoerdsma A 1976. Studies of betaaminopropionitrile in patients with scleroderma. Clin Pharmacol Therap 8: 593-602.

Kovacs EJ 1991. Fibrogenic cytokines: the role of im- mune mediators in the development of scar tissue. Immunol Today 12:17-23.

Kresina TF, He Q, Degli-Esposti S, Zern MA 1994. Gene expression of transforming growth factor beta 1 and extracellular matrix proteins in murine Schistosoma mansoni infection. Gastroenterology 107: 773-780.

Lukacs NW, Chensue SW, Streiter RM, Warmington K, Kunkel SL 1994. Inflammatory granuloma formation is mediated by TNF- $\alpha$-inducible intercellular adhesion molecules. J Immunol 152: 5883-5889.

Peacock EE, Madden JW 1966. Some studies on the effect of beta-aminopropionitrile in patients with injured flexor tendons. Surgery 66: 215-223.

Ricard-Blum S, Ville G, Grimaud JA 1992. Pyridinoline, a mature collagen cross-link, in fibrotic livers from Schistosoma mansoni-infected mice. Am J Trop Med Hyg 47: 816-820.

Roberts M, Butterworth AG, Kimani G, Kamau T, Fulford AJ, Dunne DW, Ouma JH, Sturrock RF 1993. Immunity after treatment of human schistosomiasis: association between cellular responses and resistance to reinfection. Infect Immun 61: 49844993.

Sommer P, Gleyzal C, Raccurt M, Delbourg M, Serrar M, Joazeiro P, Peyrol S, Kagan H, Trackman PC, Grimaud JA 1993. Transient expression of lysyl oxidase by liver myofibroblast in murine schistosomiasis. Lab Investig 69: 460-470.

Weinstock JV, Boros DL 1983. Organ dependent differences in composition and function observed in hepatic and intestinal granulomas isolated from mice with schistosomiasis mansoni. J Immunol 130: 418423.

Wilson RA 1990. Leaky liver, portal shunting and immunity to schistosomes. Parasitol Today 6: 354-358.

Wyler DJ 1992. Why does liver fibrosis occur in schistosomiasis? Parasitol Today 8: 277-279.

Wyler DJ 1995. Identification of a novel fibrogenic cytokine (fibrosin) in the egg granuloma. Abstract. Intern Symp. on Schistosomiasis, Salvador, BA, Brazil.

Wynn TA, Cheever AW, Jankovic D, Poindexter RW, Caspar P, Lewis FA, Sher A 1995. An IL-12-based vaccination method for preventing fibrosis induced by schistosome infection. Nature 376 (6541): 594596.

Wynn TA, Eltoum I, Cheever AW, Lewis FA, Gause WC, Sher A 1993. Analysis of cytokine mRNA expression during primary granuloma formation induced by eggs of Schistosoma mansoni. J Immunol 151: 1430-1440.

Yeowell HN, Marshall MK, Walker LC, Ha V, Pinnell SR 1994. Regulation of lysyl oxidase mRNA in dermal fibroblasts from normal donors and patients with inherited connective tissue disorders. Arch Biochem Biophys 308: 299-305. 